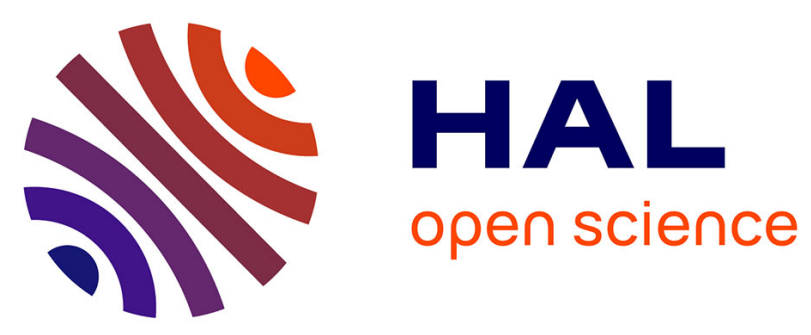

\title{
Interaction between Compliant Surfaces: How Soft Surfaces Can Reduce Friction
}

\author{
Pierre Vialar, Pascal Merzeau, Etienne Barthel, Suzanne Giasson, Carlos \\ Drummond
}

\section{- To cite this version:}

Pierre Vialar, Pascal Merzeau, Etienne Barthel, Suzanne Giasson, Carlos Drummond. Interaction between Compliant Surfaces: How Soft Surfaces Can Reduce Friction. Langmuir, In press, 10.1021/acs.langmuir.9b02384 . hal-02383883

\section{HAL Id: hal-02383883}

\section{https://hal.science/hal-02383883}

Submitted on 28 Nov 2019

HAL is a multi-disciplinary open access archive for the deposit and dissemination of scientific research documents, whether they are published or not. The documents may come from teaching and research institutions in France or abroad, or from public or private research centers.
L'archive ouverte pluridisciplinaire HAL, est destinée au dépôt et à la diffusion de documents scientifiques de niveau recherche, publiés ou non, émanant des établissements d'enseignement et de recherche français ou étrangers, des laboratoires publics ou privés. 


\section{Interaction between Compliant Surfaces: How Soft}

\section{Surfaces Can Reduce Friction}

Pierre Vialar, ${ }^{1,2}$ Pascal Merzeau ${ }^{1,2}$, Etienne Barthel ${ }^{3,4}$, Suzanne Giasson ${ }^{5 *}$ and Carlos

Drummond $^{1,2 *}$

1 CNRS, Centre de Recherche Paul Pascal (CRPP), UMR 5031, F-33600 Pessac, France

2 Université Bordeaux 1, CRPP, F-33600 Pessac, France

3 Laboratoire Sciences et Ingénierie de la Matière Molle, ESPCI Paris, CNRS, PSL University, 75005 Paris, France

4 Sorbonne Université, ESPCI Paris, Sciences et Ingénierie de la Matière Molle, 75005 Paris, France

5 Department of Chemistry and Faculty of Pharmacy, Université de Montréal, C.P. 6128, succursale Centre-Ville, Montréal, QC, Canada, H3C 3J7

Corresponding Author

*drummond@crpp-bordeaux.cnrs.fr, suzanne.giasson@umontreal.ca

ABSTRACT. We describe how a long-range repulsive interaction can surreptitiously modify the effective geometry of approaching surfaces, if compliant, with significant consequences on friction. We investigated the behavior under shear and compression of mica surfaces coated withpoly (N-isopropylacrylamide) pNIPAM-based cationic microgels. We show that local surface deformations as small as few nanometers must be considered to understand the response of such surfaces under compression and shear, in particular when the range of action of normal and friction forces are significantly different, as is often the case for macromolecular lubrication. Under these conditions, a subtle interplay between normal forces and surface compliance may significantly reduce friction increment by limiting the minimum approach of the surfaces under pressure. We found that stiffening of compressed microgels confined in the region of closest approach make it 
increasingly difficult to reduce the gap between the mica surfaces, limiting the deformation of microgels distant from the contact apex and their contribution to global friction while increasing the effective contact radius. These findings reveal a simple mechanism for robust control of lubrication: by properly tuning stiffness and geometry of the interacting bodies, for an ad hoc long range interaction, the growth of friction with applied normal load can be significantly hindered. Thus, substrate compliance is as significant as surface interaction in the design of low friction, long life systems.

KEYWORDS friction, surface forces, articular joints, lubrication, elastic deformation

\section{INTRODUCTION}

The most efficient strategy to reduce friction and wear between rubbing surfaces is to keep the surfaces away from each other. In hydrodynamic lubrication, high viscosity lubricants are commonly used to improve the normal force generated during the motion, avoiding the contact between the surfaces. This lubrication regime can be well described based on Reynold's equation. ${ }^{1}$ Under more severe conditions (e.g. at low velocities or at high applied normal force between the surfaces), the boundary lubrication regime is reached. In this regime, the separation between the surfaces is reduced to the order of few nanometers, and the friction force and wear rate increase substantially. It is this condition that often determines the lifetime of rubbing surfaces, from pistons to cartilages or articular prostheses.

A number of strategies have been developed to minimize the deleterious effects of friction; most of them are designed to improve the load bearing capacity and increase the separation between the surfaces for a given normal force. Efficient boundary lubricants based on electrostatic repulsion, ${ }^{2}$ steric and osmotic effects of swelling polymer coatings in good solvent resulting in long range interactions ${ }^{3}$ or a combination of these effects ${ }^{4,5}$ have often been investigated. The vast majority of studies on boundary lubrication aim to improve the performance of the system by increasingly sophisticated chemical design, involving delicately balanced chemical mixtures, to maximize both the cohesion of the boundary layer and the repulsive interaction under compression and shear. Remarkable solutions in this direction can be found everywhere in nature. For instance, the synovial fluid in mammalian articular joints combines a number of natural polyions to help reducing wear and frictional resistance between the rubbing cartilages. ${ }^{6}$ On the contrary, an aspect often neglected in research efforts aiming to mimic natural biolubricants is related to the mechanical properties of the supporting substrate. Cartilages are soft, with a complex organized structure. Thus, substantial deformation of the surfaces can emerge as a consequence of the weight or of the pressure generated by the shearing of the confined synovial fluid in the gap between the 
articular cartilages. In this work, we show that when the range of normal and friction forces differ significantly the friction forces between rubbing surfaces can be considerably diminished if the mechanical properties and the geometry of the surfaces are conveniently designed.

The effect of surface deformation on the description of normal interaction forces have been extensively discussed in the past by several groups. Interesting accounts were reported by Hughes and White ${ }^{7}$, Attard and Parker ${ }^{8,9}$, and Barthel. ${ }^{10}$ These authors discussed the important repercussions of surface deformation on the analysis and theoretical description of experimentally measured (static) normal forces. Here, we extend some of these ideas to the study of (dynamic) friction forces between surfaces in the presence of a long range repulsive interaction.

\section{EXPERIMENTAL SECTION}

\section{Materials}

N-Isopropylacrylamide (NIPAM), N,NMethylenebisacrylamide (MBA), aminoethyl methacrylate hydrochloride (AEMH) and 2,2'-Azobis(2-methylpropionamidine) dihydrochloride (V50) were obtained from Sigma Aldrich, and used as received. 2-(3,4-epoxy cyclohexyl) ethyl trimethoxysilane, ECHETES, was purchased from Gelest and used as received.

\section{Microgel preparation}

Microgels were synthesized by batch radical polymerization following the protocol described in reference. ${ }^{11}$ For the data reported in this work, the monomers NIPAM, MBA (crosslinking agent) and AEMH (aminated monomer which provides charged sites to stabilize the microgels and reactive sites for chemical grafting) were diluted in Milli-Q grade water (Millipore) in mass proportion 90.3/5/2.5 (1g NIPAM/50 ml). The solution was heated at $75^{\circ} \mathrm{C}$ and deoxygenated under nitrogen for 1 hour. In parallel, the initiator V50 was diluted in water $(1 \mathrm{~g} / 100 \mathrm{ml})$, heated at $70^{\circ} \mathrm{C}$ and deoxygenated for 10 minutes. After dissolution, the initiator solution was added to the monomer solution (2.1 gr V50/90.3 gr NIPAM), and the reaction was pursued at $75^{\circ} \mathrm{C}$ under continuous nitrogen flow for 4 hours. Then, the microgel dispersion was cooled down to room temperature and purified by dialysis against deionized water during 3 days, replacing the aqueous bath three time each day. Following this procedure, microgel particles of $120 \mathrm{~nm}$ hydrodynamic radius at $25^{\circ} \mathrm{C}$ in water were obtained (as determined by dynamic light scattering, Malvern Zetasizer). At room temperature, pNIPAM microgels are largely swollen with water; water volume fractions in hydrated microgel particles can exceed 0.9. ${ }^{12}$ A second set of experiments were performed with a more compliant microgel with similar chemical composition (NIPAM, MBA, AEMH and V50 in proportions 93.6/1.6/2.5/2.2) and chemical grafting method, with similar qualitative results.

\section{Chemical grafting on mica}

Cationic microgels were chemically grafted on the mica surfaces by modifying a procedure detailed before ${ }^{13}$. In brief, mica surfaces were activated by treatment with an Ar/water plasma, and coated with a layer of ECHETES. The active hydrogen in nucleophilic primary amine group (provided by the AEMH monomer) reacts with the ECHETES epoxy group to form a stable secondary amine at high yield.

Mica is a relatively inert material. However, there are $\mathrm{Si}-\mathrm{O}-\mathrm{Si}$ bonds on its surface that can be activated to form reactive silanol (Si-OH). We have used a water/Ar plasma (0.4 mbar/0.1 mbar) 
for mica activation. Ar gas breaks the $\mathrm{Si}-\mathrm{O}-\mathrm{Si}$ bonds on the surface, while water vapor oxidizes the silica atoms to form silanols, Mica surfaces were exposed to a plasma activated at $40 \mathrm{~W}$ for 5 minutes (Quorum, K1050X). After plasma treatment the surfaces were left under vacuum $\left(10^{-2}\right.$ mbar) for 5 minutes, and then they were immediately immersed in the reactive silane solution (50 $\mathrm{mM}$ ECHETES in absolute ethanol) for functionalization (silanol functions formed on the mica are unstable, and react quickly upon contact with air). Silanized mica surfaces were then rinsed with filtered ethanol to remove unreacted ECHETES, and dried at $90^{\circ} \mathrm{C}$ for 2 hours. Finally, the epoxy-modified mica surfaces were immersed in the microgel dispersion at $50^{\circ} \mathrm{C}$ for chemical grafting of the microgel particles. A typical AFM micrograph (Icon, Bruker) of a mica surface coated by the microgel particles taken in dry conditions in tapping mode is presented in Figure 1 (inset). The large surface density and uniformity of the microgel coating is apparent. Microgel particle density $c a$. 17 particles/ $\mu \mathrm{m}^{2}$ was determined by particle counting from 10 AFM micrographs taken in different points of the surfaces. This would imply a surface coverage fraction of 0.77 (neglecting microgel deformation upon adsorption; see below).

\section{Surface Forces Apparatus.}

We investigated the normal and friction forces between mica surfaces coated by poly (Nisopropylacrylamide) pNIPAM-based cationic microgel by using a Surface Forces Apparatus (SFA) modified for nanotribological studies. This technique has been amply described in the literature. ${ }^{14}$ It is commonly used to measure the interaction between mica surfaces glued on silica lenses in crossed-cylinder configuration, which is equivalent to sphere-on-a-plate if the surface separation $D$ is much smaller than the radii of curvature of the surfaces, $R$. Multiple beam interferometry (MBI) is used to measure $D$ with subnanometric resolution. ${ }^{15}$ The zero separation $(D=0)$ reported in this work is determined from the thickness of the mica surfaces measured in adhesive contact in air, and corresponds to mica-mica contact. After determination of their thickness the surfaces were removed from the SFA for microgel grafting, which was carried out ex-situ. For this reason, there is an inevitable uncertainty in the value of zero distance (smaller than $1 \mathrm{~nm}$ ), inconsequential for the data analysis.

Precisely controlled contact regions are studied in virtue of the molecular smoothness of mica. 4 contact positions were investigated with the microgel reported in this work, with quantitatively similar results. Similar results were also obtained in 6 independent experiments, with independently microgel-modified pairs of mica surfaces. Normal and lateral forces were measured from the deflection of double cantilever springs attached to the surfaces. The normal interaction $F_{N}$ was determined by displacing the position of the spring attached to the lower surface using a nanopositioner (Physik Instruments), and measuring the actual variation in the separation between the surfaces by MBI. The difference between both quantities indicates a variation in spring deflection, used to calculate $F_{N}$. To measure the lateral (friction) force, $F_{f}$, the upper surface was attached to a vertical double cantilever spring whose deflection was monitored using semiconductor strain gauges (SurForce Inc). Constant velocity, $V$, cyclic (back-and-forth) lateral motion between the surfaces was imposed by using voltage-driven bimorph strips attached to the lower surface. ${ }^{16}$

\section{RESULTS AND DISCUSSION}

The interaction between the microgel-coated mica surfaces was measured with the surfaces completely immersed in a $1 \mathrm{mM} \mathrm{NaNO}_{3}$ solution. Salt was added to enhance the screening of the 
electrostatic repulsion between the microgel-coated surfaces (the expected Debye length in the system is ca. $9.6 \mathrm{~nm}$ ). Figure 1 shows typical results for the normal $F_{N}$ and friction $F_{f}$ force between surfaces (sheared at $10 \mu \mathrm{m} / \mathrm{s}$ ). As it is commonly done, the forces are normalized by the unperturbed radius of curvature of the asperity, $R_{0}$, measured with the surfaces well separated (ca. $2 \mathrm{~cm}$ ). It is clear that the range of interaction of $F_{N}$ and $F_{f}$ are significantly different; while the onset of the repulsive osmotic force originated by the overlap of the polymer layers appears as far as $200 \mathrm{~nm}, F_{f}$ is not significant before the microgels are largely confined, at separations below 50 $\mathrm{nm}$ (the threshold of normal surface interaction, $c a .200 \mathrm{~nm}$, can be considered to be twice the average normal reach of the microgel particles, suggesting some extent of particle deformation upon grafting). Closer in, $F_{f}$ increases rapidly, attaining the same order of magnitude as the normal interaction force; however, at high applied $F_{N}$ the measured friction does not increase further. This can be clearly observed in the dependence of the measured friction with the applied normal force $F_{N}$, as presented in Figure 2. $F_{f}$ increases rapidly at low loads, but it seems to reach a maximum value at intermediate to large $F_{N}$ values. The results can also be represented in terms of the friction coefficient $\mu$, defined as $F_{f} / F_{N} . \mu$ decreases continuously at large loads, reaching values lower than 0.05 , typically reported for natural systems. ${ }^{17}$ As a reference, in absence of the microgel coating mica surfaces under load jumped into an adhesive contact, with values of static friction too large to be measured with our experimental setup $(>5 \mathrm{mN})$. The apparent saturation of $F_{f}$ with $F_{N}$ has also been observed with core-shell microgels, ${ }^{18}$ but is extremely uncommon: in the vast majority of the systems studied, $F_{f}$ increases with the applied $F_{N}$, which is ultimately accompanied by wear of the rubbing surfaces. No sign of irreversible wear was observed in this study; in particular, $F_{N}$ vs. $D$ data was similar before and after completion of the experiment, after several hours of shear.

The likely reason of the unusual behavior of $F_{f}$ with $F_{N}$ is related to the contact mechanics of the rubbing surfaces under pressure. Figure 3a shows the geometry of the gap between the mica surfaces at the different applied loads measured with an accuracy of $0.2 \mathrm{~nm}$ by Multiple Beam Interferometry. Because of the crossed-cylinder geometry of the experiment (closely corresponding to a sphere-plate geometry), it is enough to represent the separation between the surfaces $D$ as a function of the radial coordinate $X$. As can be expected, the minimum separation decreases as $F_{N}$ augments. The applied $F_{N}$ is in equilibrium with the osmotic long range repulsive force originated by the confinement of the polymeric microgels. This osmotic pressure quickly increases at shorter separations, given that the microgels are chemically grafted and cannot escape from the contact region. By decreasing the separation between the surfaces the effective stiffness of the trapped polymer layer augments. At larger separations (low $F_{N} ; D>50 \mathrm{~nm}$ ), the microgels are severely deformed, while the glue layer remain unaltered. On the contrary, at shorter separations the substrate is progressively deformed, and the geometry of the interacting surfaces is increasingly modified (Fig. 3a).

In the multilayer disposition of the SFA experiments (glass-glue-mica) it is important to consider the deformation of the different components. The compliance measured under an applied normal load will be first dominated by the mica (for extremely low loads), then by the glue and finally by the underlying silica lenses. The contact stiffness of the silica lenses can be calculated considering a semi-infinite media, as

$$
S_{\text {silica }}=2 \cdot E_{\text {silica }} \cdot a
$$

where $a$ is the radius of the contact area and $E_{\text {silica }}$ the Young modulus of the silica. ${ }^{19}$ The stiffness of a glue layer of thickness $h$ on a stiff substrate depends on its thickness as 


$$
S_{\text {glue }}=\pi \cdot \tilde{E}_{\text {glue }} \cdot \frac{a^{2}}{h}
$$

where the oedometric modulus of the glue is defined as

$$
\tilde{E}_{\text {glue }}=E_{\text {glue }} \cdot \frac{\left(1-v_{\text {glue }}\right)}{\left[\left(1-2 \cdot v_{\text {glue }}\right)\left(1+v_{\text {glue }}\right)\right]}
$$

where $E_{\text {glue }}$ and $v_{\text {glue }}$ are the Young's modulus and Poisson ratio of the epoxy glue. ${ }^{20}$ For the experimental conditions investigated in this work, contribution of mica deformation will be negligible, and $S_{\text {glue }}<S_{\text {silica }}$; hence, the deformation of the epoxy layer will be the most significant contribution. As can be observed in Figure 3, the experimentally determined load-induced deformation data can be described assuming an effective Young modulus $\mathrm{E}^{*}=7 \mathrm{GPa}$, which is dominated by the expected modulus of the epoxy glue used, and is much smaller than the modulus of silica ( $c a .75 \mathrm{GPa}$ ) or mica $(c a .170 \mathrm{GPa})$. It is important to emphasize that the contact geometry measured by MBI corresponds to the actual shape of the contact region, as the mica thickness is essentially unchanged at the low pressures used in this study.

In our experiments, the deformation of the surfaces is significant; this fact is often neglected. When the surfaces are brought in by a step $\delta$, the distance between surfaces decreases by significantly less than $\delta$, because of increasing surface deformations. The effect of surface deformation can be clearly observed if the inter-surface gap is corrected by the far-field displacement $\delta$ (displacement of the silica disk) (Figure 3b), or if the distance origin is taken as the position of closest approach between the surfaces for each normal load, $D_{0}$ (Figure $3 \mathrm{c}$ ). As can be observed in the Figure 3b-c, the aspect of the contact region is increasingly deformed at large loads, with a substantial deformation observed far from the apex. Because of the long range of the repulsive interaction, this deformation is clearly different from the Hertzian type. As a comparison, in the absence of long-range repulsion between the surfaces (e.g. in a non-adhesive Hertzian contact $^{21}$ ) a clear flat area (area of contact, $A$ ) which increases with $F_{N}$ can be identified for surfaces of similar elastic modulus $E^{*}$ under similar loads (Fig. 3e). Thus, while in a repulsive, short-range, hard contact the elastic surfaces get into intimate contact under pressure and a truly flat area of contact can be identified (Fig. 3e), surfaces interacting under a purely repulsive long-range interaction do not appear completely flat (Fig. 3a): surface deformation is evident at finite separations, but intimate surface contact (mica-mica contact in the present case) is never achieved.

For homogeneous smooth surfaces in perfect contact, it is often observed that $F_{f}$ is proportional to $A{ }^{22,23}$ Bowden and Tabor proposed that the friction between surfaces in intimate contact can be described as $F_{f}=S_{c} A$, where $S_{c}$ is the shear stress at the interface. ${ }^{24,25}$ However, even for molecularly smooth surfaces in contact, it is not always straightforward to assess what the real area of contact is, or if it is in fact meaningful to consider the concept of a real area of contact. ${ }^{25,26}$ If there is no long-range interaction between the surfaces in contact (e.g. for a Hertzian contact, Fig. 3e) $A$ can be easily identified. On the contrary, when surface forces of relatively long-range are present, the situation is more involved. Thus, it seems difficult to establish the existence of a defined contact area from the data presented in Fig. 3a. The contact profile shifted with respect to the point of closest approach (Fig. 3c) seem to suggest that above a certain load, the effective contact area between the rubbing surfaces is barely changing with $F_{N}$. If the frictional resistance between the microgel decorated surfaces is mostly determined by the interaction (i.e. collisions) 
between microgel particles on the opposite surfaces, the number of collisions (proportional to $A$ ) will be a slowly changing function of $F_{N}$, as observed experimentally (cf. Fig.2).

The effect of surface compliance will be most influential when the expected variation of the normal deformation at the point of closest approach, $\delta s$, is similar or larger than a virtual distance reduction $\delta D$ (Fig. 3f). In other words, if the additional deformation of the surfaces is similar to the applied far-field displacement (the motion imposed on the supporting cylinder) the point of closest approach of the surfaces will barely change upon increasing the applied normal force. For a repulsive interaction that increases exponentially with decreasing $D$ (as the one presented in Fig. 1), this condition will materialize at sufficiently low separations. From Hertz theory [11], a first valuation of $s$ for the purely repulsive interaction studied in this work can be estimated by

$$
S=\left(\frac{3}{2} \frac{F_{N}}{E^{*}}\right)^{\frac{2}{3}} \cdot R^{-\frac{1}{3}}
$$

where $E^{*}$ is the (unknown) reduced effective Young modulus of the mica-glue-glass multilayer composite. When $-d s / d D=-d s / d F_{N} d F_{N} / d D>1$, the contact deformation due to the repulsive force will strongly limit any further reduction of the minimum gap between the surfaces under further compression. For the system investigated in this work, this condition is fulfilled when $D<$ $c a .35 \mathrm{~nm}$. A more precise description of the geometry of the contact region under compression in presence of the long-range repulsion can be attained by using the numerical method outlined by Barthel $^{10}$ (continuous lines in Figure 3a-c). Quantitative agreement between measured and calculated geometry is obtained by adjusting $E^{*}$ of the contact as the only fitting parameter, and using the measured normal force profile (Fig. 1) and the non-deformed surface radius $R_{0}$ as inputs. Thus, the calculated data match closely not only the contact geometry but also the experimentally determined $F_{N}$ force profile. It is important to emphasize that $F_{N}(D)$ measured with compliant surfaces will not be the same as the interaction between non-deformable (infinitely stiff) surfaces, in particular under large compressions (see below). The quality of the fits can be better assessed from the surface force induced elastic deformation of the interacting surfaces, $\Delta D(X)$ (Fig. 3d); as can be observed in this figure, the calculated deformation agrees well with the measured data, which corresponds to the largest load presented in Figures 3a-c.

The potential effect of long range interaction on surface forces measurement has been anticipated before. Hughes and White ${ }^{7}$ and Barthel ${ }^{10}$ have investigated the possible influence of a long range repulsive interaction on the force of interaction between finite compliance surfaces. As an illustration, we have calculated the normal force vs distance profile expected for the case of surfaces interacting through a single exponential repulsive force of the same order of magnitude as the one reported in Figure 1, for contacts of different reduced Young modulus $E^{*}$. As can be observed in Figure 4a, for an infinitely stiff contact $F_{N}$ is a purely exponential decay function of $D$, as expected. On the contrary, for more compliant surfaces, the measured $F_{N} / R_{0}$ (repulsive force normalized by the unperturbed radius) deviates from the exponential law; this deviation is noticeable at larger separations for smaller values of $E^{*}$. This deviation is caused by the elastic deformation of the surfaces, which needs to be considered in the description of the system, in addition to the surface forces and the externally applied load. Similar results have been described in the past by different groups ${ }^{7-10}$. 
The more compliant the surfaces, the more efficient the repulsive force in keeping the surfaces apart. As the surface forces become increasingly repulsive, the deformable elastic surface becomes the weakest link of the chain. It is interesting to examine the geometry of the interacting surfaces. Two examples are presented in Figure $4 \mathrm{~b}$ for different values of $E^{*}$. As expected, the geometry of the softer contact is more affected by the applied $F_{N}$. Less intuitively, the softer surfaces will also end up more separated for similarly applied $F_{N}$. In other words, for the same load, both the penetration (far field displacement) and the distance of closest approach will be larger for softer surfaces. If the friction force takes up at a distance smaller than the range of this repulsive interaction, the separation between surfaces can be robustly kept larger than this threshold, and friction will be strongly limited (cf. Figure 1), limiting the global $F_{f}$. On the contrary, if the final separation is significantly lower than this characteristic distance, the deformation of the surfaces implies an augmented effective area of interaction $A$ and the concomitant increase of $F_{f}$ with $F_{N}$, as is commonly observed.

The limited growth of $F_{f}$ with $F_{N}$ discussed in this work has purely static causes: it is a consequence of the compliant surfaces been kept apart due to surface forces. It is not associated with the presence of repulsive dynamic normal (lift) forces (e.g. elastohydrodynamic forces), which are also related to the finite compliance of the rubbing surfaces and can also play a role under shear, or to the recently reported elastic deformation of surfaces under oscillating drainage flow. ${ }^{27}$ As can be inferred from these results, even a rather small deformation of the contact region at moderately low applied loads can have a significant influence on the global tribological behavior of the system, because the effective radius (and hence the load bearing capacity) increases significantly. Several important consequences emerge from these results. First, it may be necessary to reconsider the description and analysis of experimental results including the influence of the elastic deformation of the surfaces, often ignored by the community studying surface forces. Commonly, measured force profiles are reported as $F / 2 \pi R_{0}$, which, following the Derjaguin approximation, ${ }^{28}$ is considered to be numerically equal to the interaction energy per unit area between the two planar walls undergoing the same interaction as the curved objects, $E(D)$. However, this is only valid in the absence of deformation of the interacting surfaces. Otherwise, to rigorously relate the measured force to $E(D)$, it is necessary to consider the elastic deformation of the surfaces; it may be computationally costly, but it is the correct way to obtain a good assessment of the interaction between non-deformed surfaces, which is better adapted for comparison with theoretical descriptions. In addition, the results presented here, and in particular the apparent saturation of $F_{f}$ with increasing $F_{N}$, hint for strategies to limit the effect of friction and wear on compliant systems based on tuning surface morphology, surface compliance or the range of the repulsive interaction forces. If the range of friction and normal interaction are sufficiently dissimilar - as often observed for systems lubricated by macromolecular coatings ${ }^{29,30}$ - it is possible to minimize the friction coefficient by restricting the approach of the rubbing surfaces while limiting the growth of the effective area of interaction under shear by adequately tuning $E^{*}$ and the asperity size $R$.

\section{CONCLUSIONS}

Convex surfaces undergoing a long-range repulsive interaction —as often observed with macromolecular coatings - can get significantly deformed at finite separations. However, as the deformed surfaces are not in intimate contact, no flat region of contact can be clearly identified. If the size of the contact and the stiffness of the protecting layer are adequately chosen, the deformation of the surfaces severely limits surface approach, possibly restricting the growth of 
friction with compression. This scenario becomes more significant when the effective distancerange of normal and frictional forces are significantly different. 


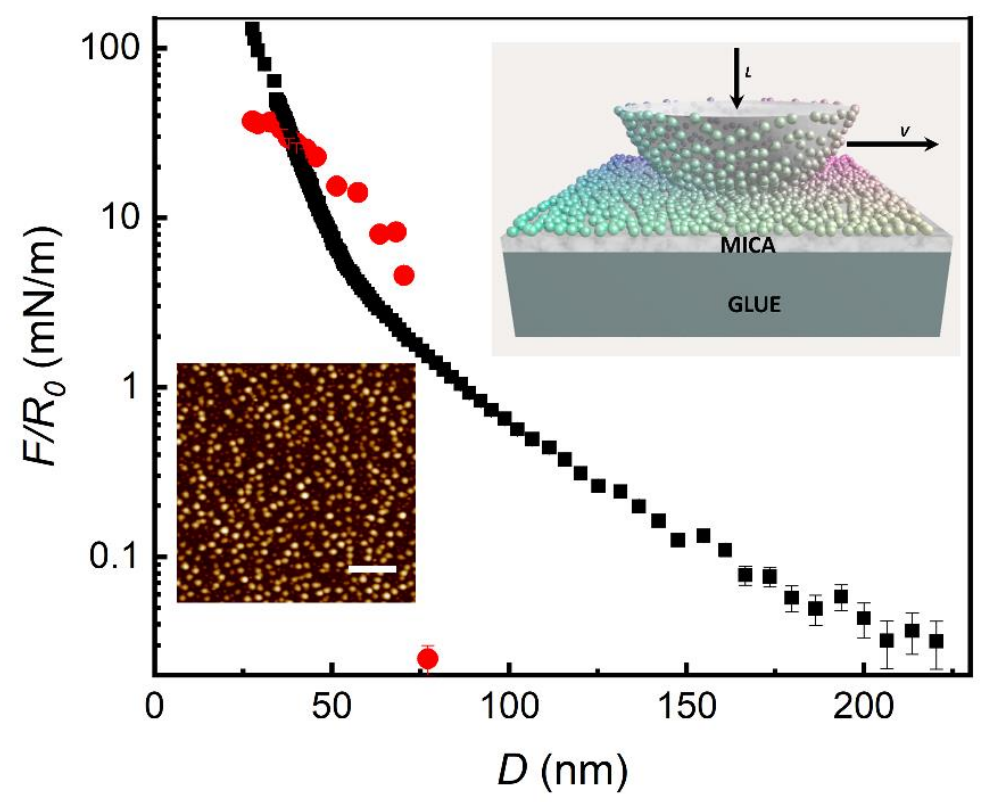

Figure 1. Interaction forces (normalized by the non-deformed radius of curvature of the surfaces, $R_{0}=2.09 \mathrm{~cm}$ ) vs surface separation $D$ between mica surfaces coated with a chemically grafted layer of pNIPAM microgels immersed in a solution of $1 \mathrm{mM} \mathrm{NaNO}_{3}$. Black squares: normal force, $F_{N}$. Red circles: friction force $F_{f}$ measured at $V 10 \mu \mathrm{m} / \mathrm{s}$. The normal force profile was measured during quasi-static approach. Lower inset: AFM tapping mode micrograph measured in air (ex-situ) of a dried microgel-coated mica surface, after the SFA experiments. Scale bar $1 \mu \mathrm{m}$

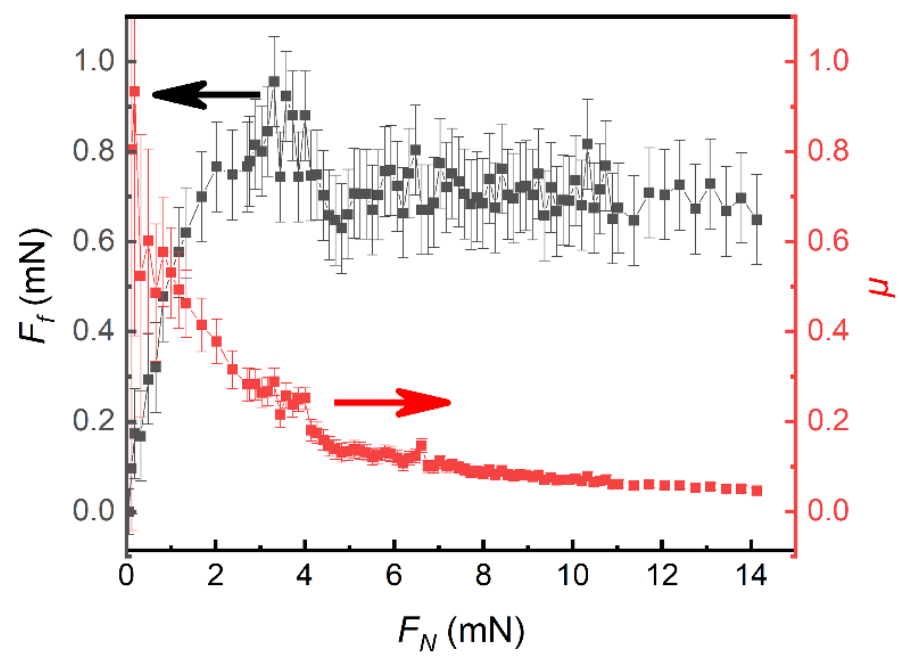

Figure 2. Load-dependence of the friction force $F_{f}$ and friction coefficient $\mu$ between mica surfaces coated with a chemically grafted layer of pNIPAM microgels immersed in a solution of $1 \mathrm{mM}$ $\mathrm{NaNO}_{3}$. 

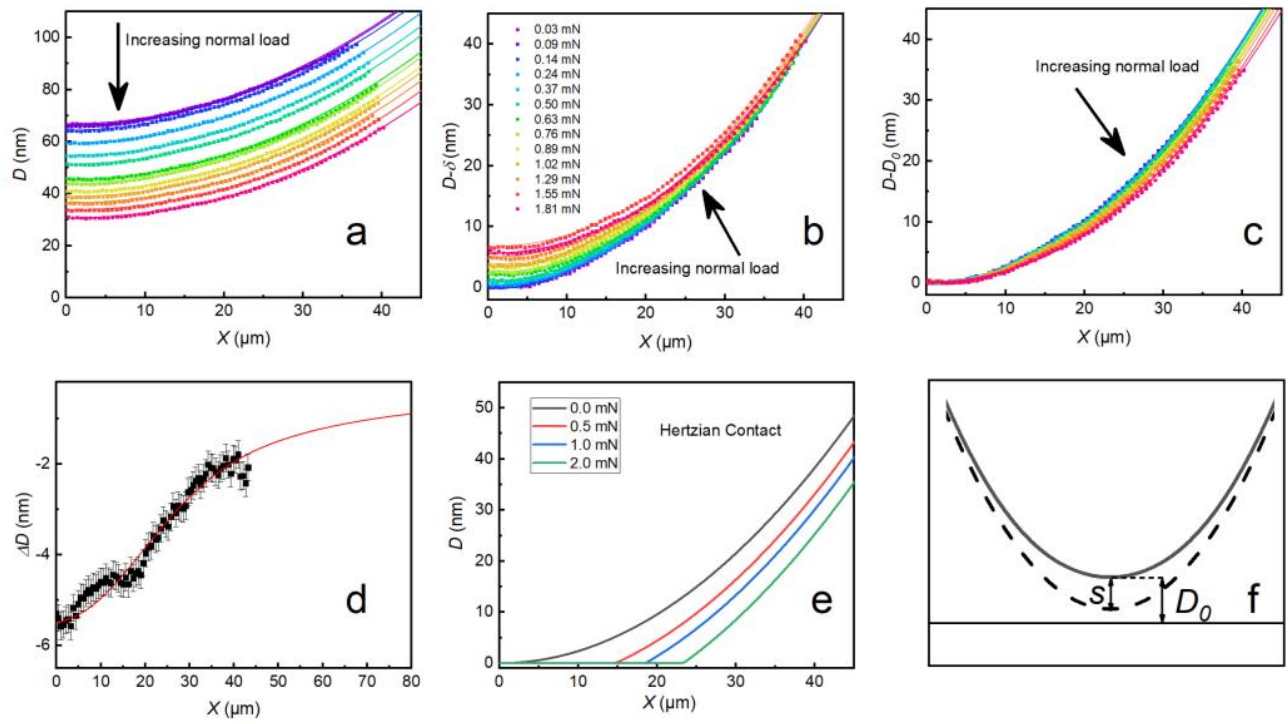

Figure 3. Geometry of the interacting surfaces under compression. a) Measured (points) and fitted (lines) data for different applied normal loads, as indicated in panel b). The fitted geometries were determined for an effective Young modulus $E^{*}=7 \mathrm{GPa}$, which gives the best adjustment to the ensemble of the measured data. b) and c) Same data as in a), shifted by the (b) far-field displacement, $\delta$, or (c) the point of closest approach, $D_{0}$. d) Difference between the deformed and non-deformed geometry, for the measured (points) and fitted (red line) data; normal load L=1.81 $\mathrm{mN}$. e) Expected surface geometry for a Hertzian contact of $E^{*}=7 \mathrm{GPa}$, for the values of normal load indicated in the inset. f) Illustration of the geometry of the system, showing the deformation $s$ at the point of closest approach; deformed (continuous line) vs non-deformed (dashed line) geometry.
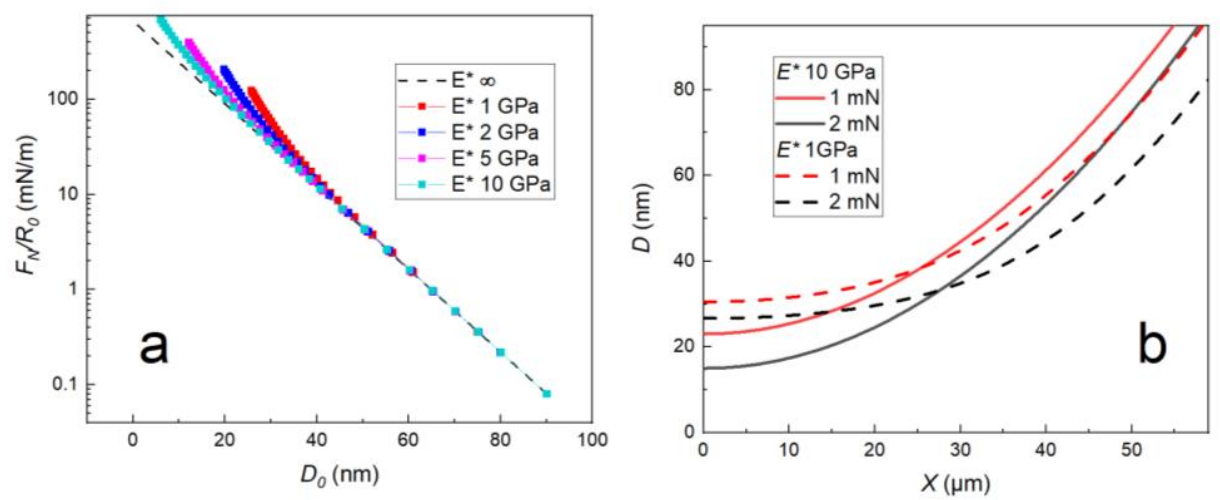

Figure 4. a) Normal force of interaction normalized by the non-deformed asperity radii for contacts of different effective Young Modulus $E^{*}$, considering that the non-deformed surfaces interact following a single-exponential decay force (characteristic length $8 \mathrm{~nm}$ ). b) Corresponding contact geometry for different values of $E^{*}$ and applied loads. Non-deformed asperity radii $R_{0}=2$ cm 


\section{Notes}

The authors declare no competing financial interests.

\section{ACKNOWLEDGMENT}

Financial support from the Natural Sciences and Engineering Research Council of Canada, Groupe de Recherche Universitaire sur le Médicament and Centre de Recherche sur les Matériaux AutoAssemblés are acknowledged.

\section{REFERENCES}

(1) Reynolds, O. On the Theory of Lubrication and Its Application to Mr. Beauchamp Tower's Experiments, Including an Experimental Determination of the Viscosity of Olive Oil. Philos. Trans. R. Soc. London 1886, 177 (0), 157-234.

(2) Richetti, P.; Drummond, C.; Israelachvili, J.; In, M.; Zana, R. Inverted Stick-Slip Friction. Europhys. Lett. 2001, 55 (5), 653-659.

(3) Klein, J. Shear, Friction, and Lubrication Forces Between Polymer-Bearing Surfaces. Annu. Rev. Mater. Sci. 1996, 26 (1), 581-612.

(4) Chen, M.; Briscoe, W. H.; Armes, S. P.; Klein, J. Lubrication at Physiological Pressures by Polyzwitterionic Brushes. Science 2009, 323 (5922), 1698-1701.

(5) Raviv, U.; Giasson, S.; Kampf, N.; Gohy, J.-F.; Jérôme, R.; Klein, J. Lubrication by Charged Polymers. Nature 2003, 425 (6954), 163-165.

(6) Jahn, S.; Seror, J.; Klein, J. Lubrication of Articular Cartilage. Annu. Rev. Biomed. Eng. 2016, 18 (1), 235-258.

(7) Hughes, B. D.; White, L. R. Implications of Elastic Deformation on the Direct Measurement of Surface Forces. J. Chem. Soc. Faraday Trans. 1 Phys. Chem. Condens. Phases 1980, 76, 963.

(8) Parker, J. L.; Attard, P. Deformation of Surfaces Due to Surface Forces. J. Phys. Chem. 1992, 96 (25), 10398-10405.

(9) Attard, P.; Parker, J. L. Deformation and Adhesion of Elastic Bodies in Contact. Phys. Rev. A 1992, 46 (12), 7959-7971.

(10) Barthel, E. Surface Deformation, Spring Stiffness and the Measurement of Solvation Forces. Thin Solid Films 1998, 330 (1), 27-33.

(11) López-León, T.; Ortega-Vinuesa, J. L.; Bastos-González, D.; Elaïssari, A. Cationic and Anionic Poly( N -Isopropylacrylamide) Based Submicron Gel Particles: Electrokinetic Properties and Colloidal Stability. J. Phys. Chem. B 2006, 110 (10), 4629-4636. 
(12) Yang, M.; Liu, C.; Zhao, K. Concentration Dependent Phase Behavior and Collapse Dynamics of PNIPAM Microgel by Dielectric Relaxation. Phys. Chem. Chem. Phys. 2017, $19(23), 15433-15443$.

(13) Banquy, X.; Zhu, X. X.; Giasson, S. Mechanical and Frictional Properties of Nanoparticle Monolayers Grafted on Functionalized Mica Substrates. J. Phys. Chem. B 2008, 112 (39), $12208-12216$.

(14) Israelachvili, J.; Min, Y.; Akbulut, M.; Alig, A.; Carver, G.; Greene, W.; Kristiansen, K.; Meyer, E.; Pesika, N.; Rosenberg, K.; Zeng, H. Recent Advances in the Surface Forces Apparatus (SFA) Technique. Reports Prog. Phys. 2010, 73 (3), 036601.

(15) Israelachvili, J. N.; Physics, S. Thin Film Studies Using Multiple-Beam Interferometry. $J$. Colloid Interface Sci. 1973, 44 (2), 259-272.

(16) Luengo, G.; Schmitt, F.-J.; Hill, R.; Israelachvili, J. Thin Film Rheology and Tribology of Confined Polymer Melts: Contrasts with Bulk Properties. Macromolecules 1997, 30 (8), 2482-2494.

(17) Dowson, D. Paper 12: Modes of Lubrication in Human Joints. Proc. Inst. Mech. Eng. Conf. Proc. 1966, 181 (10), 45-54.

(18) Giraud, L.; Bazin, G.; Giasson, S. Lubrication with Soft and Hard Two-Dimensional Colloidal Arrays. Langmuir 2017, 33 (15), 3610-3623.

(19) Sridhar, I.; Johnson, K. L.; Fleck, N. A. Adhesion Mechanics of the Surface Force Apparatus. J. Phys. D. Appl. Phys. 1997, 30 (12), 1710-1719.

(20) Gacoin, E.; Fretigny, C.; Chateauminois, A.; Perriot, A.; Barthel, E. Measurement of the Mechanical Properties of Thin Films Mechanically Confined within Contacts. Tribol. Lett. 2006, 21 (3), 245-252.

(21) Johnson, K. L. Contact Mechanics; Cambridge University Press: Cambridge, 1985.

(22) Drummond, C.; Israelachvili, J.; Richetti, P. Friction between Two Weakly Adhering Boundary Lubricated Surfaces in Water. Phys. Rev. E 2003, 67 (6), 066110.

(23) Carpick, R. W.; Salmeron, M. Scratching the Surface: Fundamental Investigations of Tribology with Atomic Force Microscopy. Chem. Rev. 1997, 97 (4), 1163-1194.

(24) F P Bowden \& D Tabor. Friction and Lubrication; Methuen \& Co, Ed.; London, 1967.

(25) Homola, A. M.; Israelachvili, J. N.; McGuiggan, P. M.; Gee, M. L. Fundamental Experimental Studies in Tribology: The Transition from "Interfacial" Friction of Undamaged Molecularly Smooth Surfaces to "Normal" Friction with Wear. Wear 1990, $136(1), 65-83$.

(26) Berman, A.; Drummond, C.; Israelachvili, J. Amontons ' Law at the Molecular Level. 
Tribol. Lett. 1998, 4 (2), 95-101.

(27) Villey, R.; Martinot, E.; Cottin-Bizonne, C.; Phaner-Goutorbe, M.; Léger, L.; Restagno, F.; Charlaix, E. Effect of Surface Elasticity on the Rheology of Nanometric Liquids. Phys. Rev. Lett. 2013, 111 (21), 1-5.

(28) Derjaguin, B. Untersuchungen Über Die Reibung Und Adhäsion, IV. Colloid J. 1934, 69 (2), 155-164.

(29) Klein, J.; Kumacheva, E.; Mahalu, D.; Perahia, D.; Fetters, L. J. Reduction of Frictional Forces between Solid Surfaces Bearing Polymer Brushes. Nature 1994, 370 (6491), 634636.

(30) Bouchet, A. S.; Cazeneuve, C.; Baghdadli, N.; Luengo, G. S.; Drummond, C. Experimental Study and Modeling of Boundary Lubricant Polyelectrolyte Films. Macromolecules 2015, 48 (7), 2244-2253. 